\title{
Evaluation of peripheral nodal recurrence in patients with endometrial cancer
}

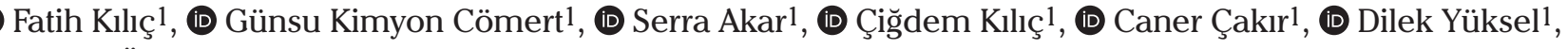 \\ (D) Mehmet Ünsal1 ${ }^{1}$ (D) Nedim Tokgözoğlu², (D) Salih Taşkın³, (D) Tolga Taşcı², (D) Osman Türkmen¹, (D) Fırat Ortaç3, \\ (D) Taner Turan 1 \\ 1Department of Gynecologic Oncology, University of Health Sciences Turkey, Etlik Zübeyde Hanım Women's Health \\ Training and Research Hospital, Ankara, Turkey \\ ${ }^{2}$ Clinic of Gynecologic Oncology, Okmeydanı Training and Research Hospital, İstanbul, Turkey \\ 3Department of Gynecologic Oncology, Ankara University Faculty of Medicine, Ankara, Turkey
}

\section{Abstract}

Objective: To evaluate the clinico-pathological patient features, prognostic factors, treatment options and outcomes of peripheral nodal recurrence (PNR) of endometrial cancer (EC).

Material and Methods: The data of nine patients with PNR of EC from two institutions were reviewed. The electronic literature was reviewed from 1972 to May 2018 to identify articles about PNR in EC. Finally, 42 cases were evaluated.

Results: Nineteen (45.2\%) patients were initially diagnosed with either stage I or II disease, whereas 20 (47.7\%) patients had stage III or IV disease while the stages were not reported in three (7.1\%). PNR developed as the first recurrence in 40 (95.2\%) patients and as the second recurrence in $2(4.8 \%)$ patients. Isolated PNR appeared in 35 (83.3\%). Seven (16.7\%) had PNR coexisting with multiple other sites of tumoral involvement. In the entire cohort, the 5-year and 10-year post-recurrence survival (PRS) were both 78\%. Only the presence of distant hematogenous metastasis concurrent with PNR was significantly related to poor PRS $(\mathrm{p}=0.005)$. Among patients with isolated PNR, those who had surgery had $30 \%$ greater 5 -year PRS than those treated without surgery, but this difference was not significant ( $80 \%$ vs 50\%; p $>0.05$ ).

Conclusion: A concurrent distant hematogenous metastasis was the only factor related to poor survival. A wide range of therapies exists for PNR but none of the therapies appear to be more advantageous than another. However, surgery as a component of treatment can render a survival advantage for patients who have isolated PNR. (J Turk Ger Gynecol Assoc 2022; 23: 38-50)

Keywords: Endometrial cancer, lymphatic failure, peripheral nodal recurrence, survival, treatment

Received: 25 April, 2021 Accepted: 06 June, 2021

\section{Introduction}

Endometrial cancer (EC) is the most common gynecological malignancy (1). Although EC has a high disease-free survival rate, its recurrence rate is $13-16 \%(2,3)$. EC usually recurs locally in the pelvis or vaginal cuff (4). The lymphatic failure in EC appears mostly in specific retroperitoneal lymph nodes, such as the pelvic and para-aortic nodes $(3,5)$. Therefore, many studies have focused on the prognostic factors and treatment options of these frequently encountered recurrence sites
(5-7). Various atypical recurrence sites have been reported (8). Peripheral nodal recurrence (PNR) is one of the rare failure patterns of EC. Due to its infrequency, it is important to detect patients who are at high risk for peripheral lymphatic failure. Treatment options range from local surgical excision to pelvic exenteration, chemotherapy, radiotherapy and palliative therapy (9-11). Furthermore, the limited information on PNR in EC is based solely on cases from the literature. Therefore, PNR treatment options in EC remain unclear. 
In the current study, a case series of PNR from EC is presented. The aim of this study was to evaluate the clinico-pathological patient features, prognostic factors, treatment choices, and outcomes of PNR in EC.

\section{Material and Methods}

Data of 1,345 patients with epithelial EC who underwent at least a hysterectomy and bilateral salpingo-oophorectomy in our gynecological-oncology clinic between January 1993 and May 2013 were evaluated. These cases were assessed for the presence of PNR, which was defined as the presence of involved lymph nodes outside the abdominal cavity (except for the mediastinal lymph nodes) in cases with at least a onemonth disease-free interval (DFI) following complete response to treatment before PNR. Patients who had a sarcomatous component identified in their histopathological examination or whose peripheral nodal involvement appeared without at least a one-month DFI were excluded. Recurrence developed in 162 of 1,345 cases with epithelial EC. The rate of PNR was 4.9\% (8/162) among patients who developed all types of recurrences from epithelial EC. These eight patients from the first institution were added to the study group. One patient from the second participating institution who had PNR was also included (12). Thus, a study group was formed with a total of nine patients from two institutions. The University of Health Sciences Turkey, Etlik Zübeyde Hanım Women's Health Training and Research Hospital Institutional Committee has approved the study protocol (approval number: 47502, date: 25.06.2018). All patients signed an informed consent that allows the institution to use their clinical data.

\section{Literature review}

A systematic review of the medical literature was conducted to identify articles about PNR after initial treatment of EC. The electronic literature search was reviewed from 1972 to May 2018 using PubMed/MEDLINE for English language abstracts. The search included the following medical subject headings or keywords: "distant" or "peripheral" or "unusual" or "supraclavicular" or "inguinal" or "neck" or "axillar" or "jugular" lymph node recurrence of EC. After the completion of the search, 29 articles were found. Subsequently, 17 articles were excluded from the study for reasons that are presented in detail in the research chart (Figure 1). In four of the excluded articles, only the locations of the distant lymph nodes were detailed and the distribution of those were: cervical and

\section{7 articles were excluded, because of}

- Definition of the type of the distant recurrence is unknown: 1

- Review: 2

- Results of the imaging: 1

- Distant lymph node involvement at initial presentation (not recurrence): 6

- Not detailed patients with distant lymph node recurrence case by case: 4

- Recurrence of cervical cancer or simultaneous endometrial and ovarian cancer: 2

- Not achievable full text and abstract: 1
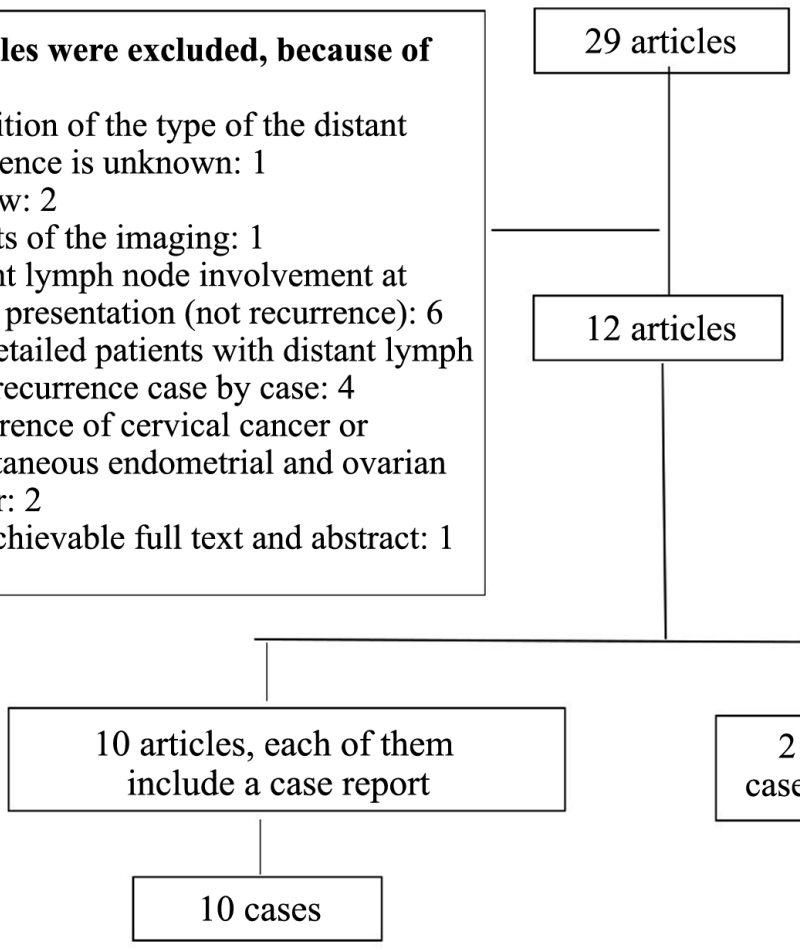

2 articles, each of them include $2 \leq$ cases $(n=33)$ and our 9 cases $=42$ cases

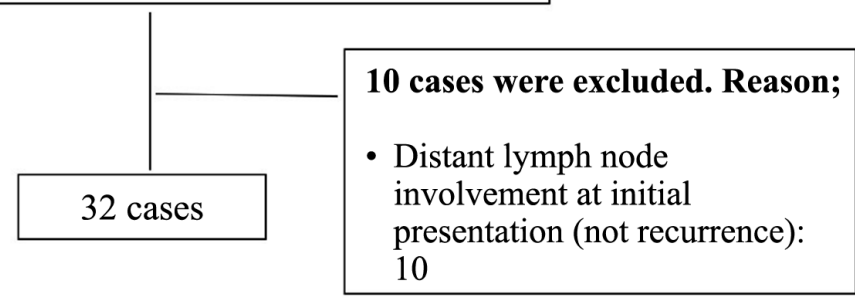

Figure 1. Chart showing details of the literature review 
supraclavicular nodes, 5 cases (13); inguinal nodes, 5 cases (13-15); cervical nodes, 5 cases (14); supraclavicular nodes, 2 cases (16); subclavian nodes, 2 cases (14); and axillary lymph nodes, 1 case (16). Therefore, only the frequency of involved nodes for these cases from the four articles was included in the analysis. Cases $(n=43)$ from the remaining 12 articles were evaluated comprehensively. Ten of the eleven cases with peripheral nodal involvement, reported in one article (17) were excluded because they had peripheral nodal involvement at initial presentation (not at recurrence). The follow-up time and end status of a case that had been previously published about PNR of EC was updated (12). Finally, we evaluated a total of 42 cases, including our case series of nine patients.

\section{Data evaluation}

Disease recurrence involving the peripheral lymph nodes alone was defined as isolated PNR. Recurrence, which developed in any other location in conjunction with peripheral lymph nodes was defined as PNR with multiple involved sites. Patients were staged according to the 2009 International Federation of Gynecology and Obstetrics (FIGO) criteria (18). Therefore, stages of patients were updated for articles that were published before 2009, if the histopathological findings were available. Tumor size was defined as the largest tumor diameter for a recurrent tumor. Tumors with undifferentiated, clear cell and serous histology were accepted as grade 3 disease. DFI was described as the time period from initial treatment to PNR for patients with the first recurrence and from treatment before PNR to appearance of PNR for patients who had a secondary recurrence. The period from PNR to last patient visit or patient death was defined as post-recurrence survival (PRS). The follow-up time was defined as the interval between initial treatment to death or the last contact with the patient. Involved cervical lymph nodes included PNR that was described as neck, jugular, or cervical in articles from the medical literature. Subclavian lymph node involvement was classified as supraclavicular lymph node involvement.

Patients with suspected PNR were evaluated by clinical examination and radiological imaging methods. Subsequently, the diagnosis of PNR was made based on these findings. Radiological imaging was evaluated by a radiologist. Suspicious peripheral lymph nodes were biopsied. Management of PNR was directed by the institutional tumor board.

Patients who had a complete clinical response after treatment for recurrence were followed-up at three-month intervals for the first two years, at six-month intervals for the next three years, and annually thereafter. Pelvic examination, complete blood count, blood chemistry and abdominopelvic ultrasonography were performed as follow-up monitoring. Chest X-ray was performed yearly unless clinical suspicion indicated otherwise.
Abdominal and/or thoracic computed tomography were used when required. Although not routinely used, CA-125 levels were utilized for follow-up.

\section{Statistical analysis}

SPSS, version 20.0 (SPSS Inc., Chicago, IL, USA) was used for statistical analysis. Descriptive statistics were expressed as mean \pm standard deviation or median (minimummaximum) for continuous variables and number/percentage for categorical variables. The Kaplan-Meier method was used for the assessment of survival outcomes. Multivariate analysis was performed using a Cox proportional hazards model. All variables with a $\mathrm{p}<0.25$ in univariate analysis were included in the multivariate analysis. Survival curves were compared using the log-rank test. A p-value less than 0.05 were considered to be statistically significant.

\section{Results}

The median (range) age of the study group was 60 (45-75) years. The histological types were endometrioid adenocarcinoma in $13(31 \%)$, clear cell adenocarcinoma in $3(7.1 \%)$, and mixed cell adenocarcinoma in 1 (2.4\%) patient. Mixed cell adenocarcinoma was composed of grade 3 endometrioid adenocarcinoma with 25\% mucinous differentiation and $15 \%$ clear cell adenocarcinoma. The type of adenocarcinoma was not specified in 22 patients. The differentiation of endometrioid adenocarcinoma was FIGO grade 1 in 7 patients, grade 2 in 3 patients, and grade 3 in 3 patients. In 22 patients, the grade was classified according to the 1988 Broder's classification (Table 1) (19). Distribution of the 2009 FIGO stages was as follows; stage 1, 17 (40.5\%) patients; stage 3, 15 patients (35.8\%); and stage 4,5 patients $(11.9 \%)$. The stages of the two patients (4.8\%) with stage 2 disease could not be updated according to the 2009 FIGO criteria because of the absence of information on the type of cervical involvement. The stage was unknown in three patients. Three patients had a history of unopposed estrogen exposure (20) breast cancer (21), and rectal cancer (11), respectively. The clinico-pathological findings of the entire cohort are shown in Table 1, 2 .

PNR developed as the first recurrence in 40 (95.2\%) patients, while in 2 (4.8\%) patients it appeared as the second recurrence. The median DFI was 15 months, ranging between 2 and 276 months. The sites of PNR reported in the four excluded articles were: inguinal lymph nodes in 26 (41.9\%); supraclavicular lymph nodes in 22 (35.5\%); cervical lymph nodes in 15 (24.2\%); and axillary lymph nodes in 5 (8.1\%). The median (range) diameter of the recurrent tumor was $3.75(2-10) \mathrm{cm}$. Isolated PNR occurred in 35 (83.3\%) patients. Seven (16.7\%) had PNR with multiple involved sites. Other sites associated with PNR were the vagina including the peri-urethral area $(n=1)$; pelvis 


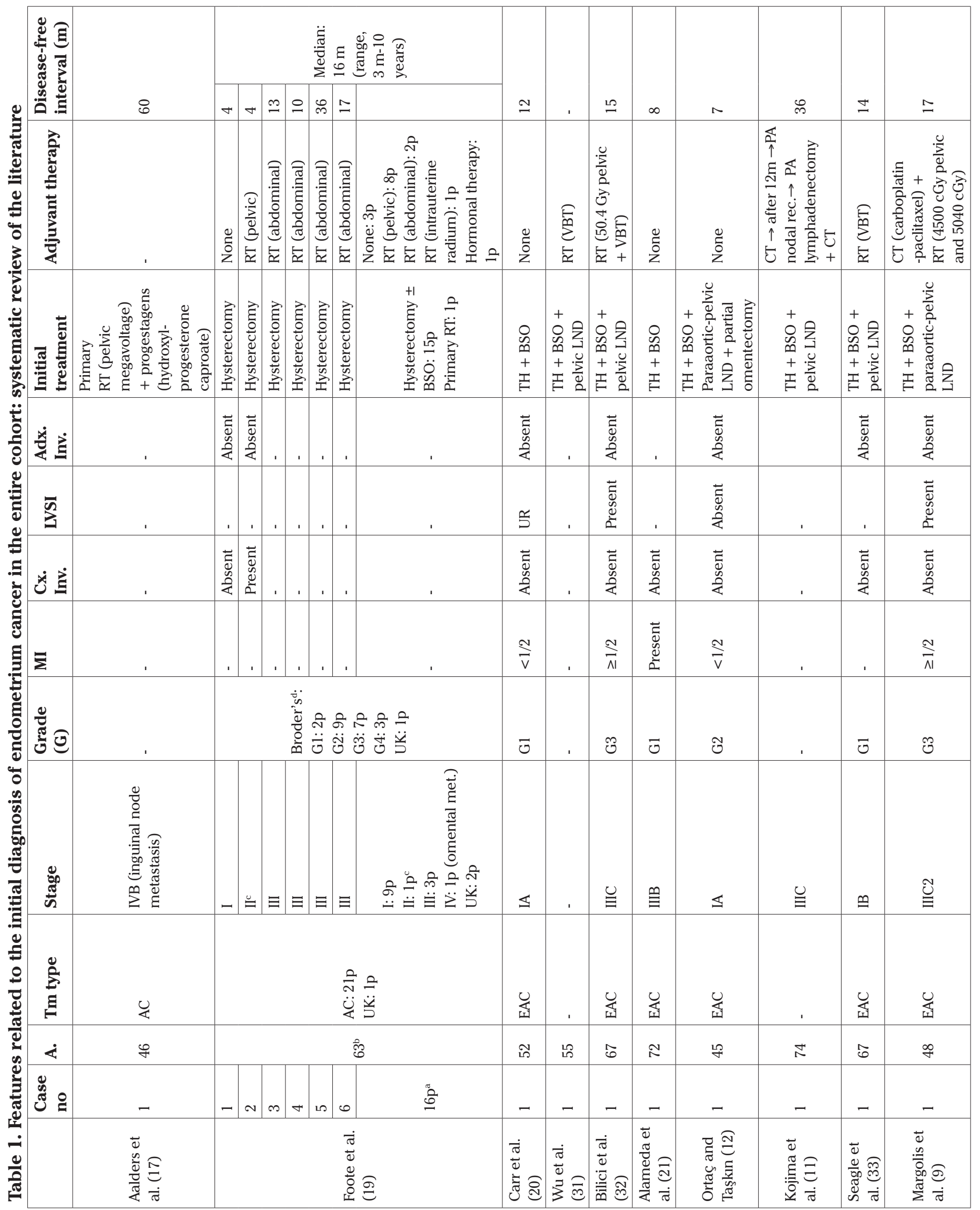




\begin{tabular}{|c|c|c|c|c|c|c|c|c|c|c|c|c|}
\hline 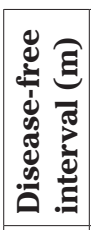 & $\stackrel{0}{0}$ & 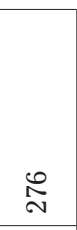 & 迌 & $\stackrel{\infty}{\infty}$ & مL & $\infty$ & ஜి & $\infty$ & 은 & ๙ & $\sim$ & 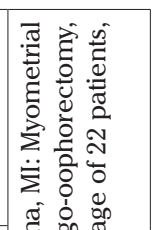 \\
\hline 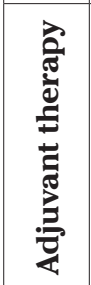 & 苋 & 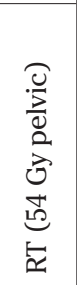 & 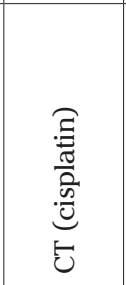 & 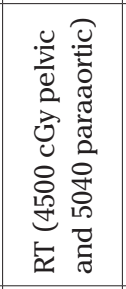 & 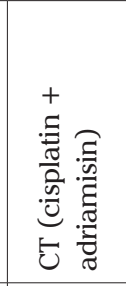 & 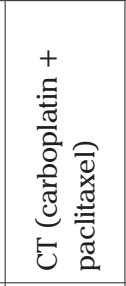 & $\stackrel{0}{\check{z}}$ & 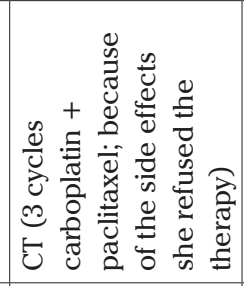 & $\underline{\underline{x}}$ & 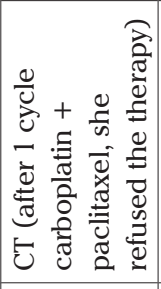 & 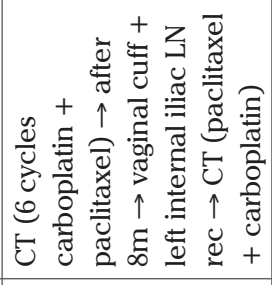 & 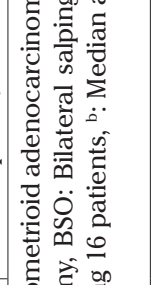 \\
\hline 焉 & $\begin{array}{l}0 \\
\infty \\
\infty \\
+ \\
I \\
\end{array}$ & $\begin{array}{l}0 \\
\infty \\
+ \\
+ \\
I\end{array}$ & 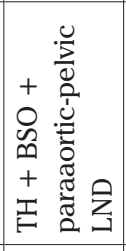 & 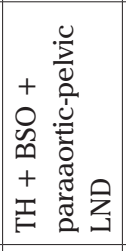 & 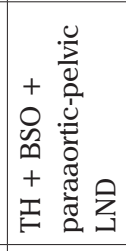 & 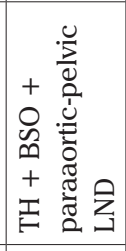 & 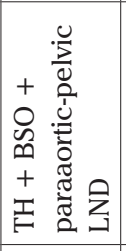 & 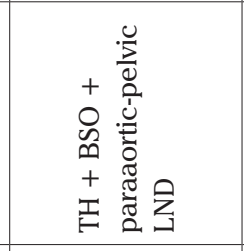 & 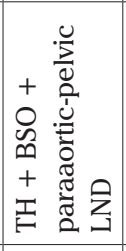 & 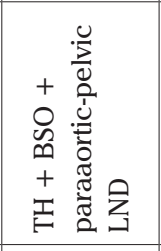 & 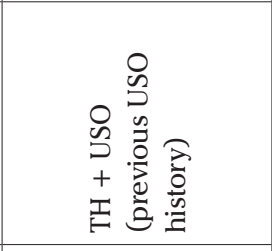 & 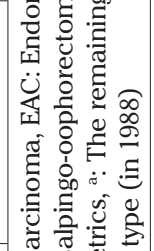 \\
\hline 运完 & 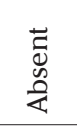 & & $\begin{array}{l}\overrightarrow{0} \\
\text { D. } \\
\text { 定 }\end{array}$ & $\begin{array}{l}\overrightarrow{0} \\
\text { D } \\
\text { 完 }\end{array}$ & $\begin{array}{l}\overrightarrow{D_{0}} \\
\text { D. } \\
\text { 定 }\end{array}$ & 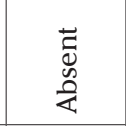 & $\begin{array}{l}\overrightarrow{0} \\
\text { D. } \\
\text { 完 }\end{array}$ & 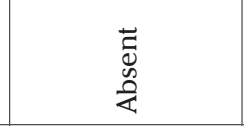 & 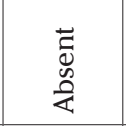 & 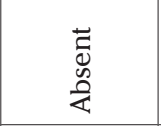 & 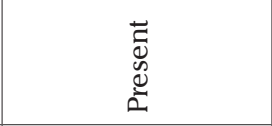 & 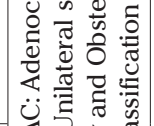 \\
\hline $\bar{z}$ & 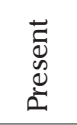 & 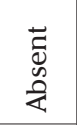 & & . & , & 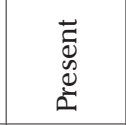 & 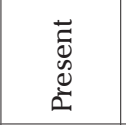 & . & 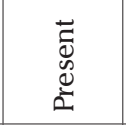 & 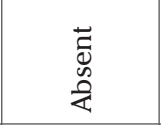 & 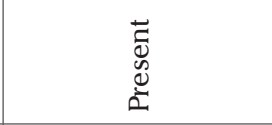 & 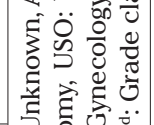 \\
\hline$\dot{u} \dot{\vec{g}}$ & 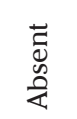 & $\begin{array}{l}\overrightarrow{0} \\
\bar{d} \\
\vec{\alpha}\end{array}$ & $\begin{array}{l}\overrightarrow{0} \\
\text { D. } \\
\text { 定 }\end{array}$ & $\begin{array}{l}\overrightarrow{0} \\
\text { D } \\
\text { 完 }\end{array}$ & 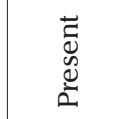 & 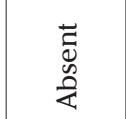 & $\begin{array}{l}\overrightarrow{\tilde{U}} \\
\text { 足 } \\
\text { \& }\end{array}$ & 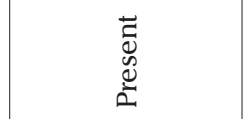 & $\begin{array}{l}\overrightarrow{\tilde{U}} \\
\text { 足 } \\
\text { \& }\end{array}$ & $\begin{array}{l}\overrightarrow{\tilde{U}} \\
\text { 足 } \\
\text { \& }\end{array}$ & 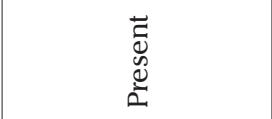 & 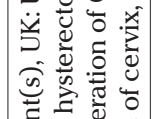 \\
\hline$\Sigma$ & 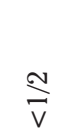 & $\stackrel{\mathcal{N}}{\vec{V}}$ & 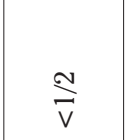 & $\stackrel{N}{N}$ & $\underset{\Lambda}{\stackrel{N}{N}}$ & $\underset{\Lambda \Lambda}{\stackrel{N}{N}}$ & $\stackrel{\sim}{\vec{v}}$ & 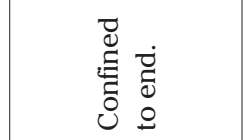 & $\underset{\Lambda}{\stackrel{N}{N}}$ & $\stackrel{N}{N}$ & $\underset{\Lambda}{N}$ & 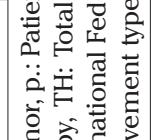 \\
\hline قृ & $\mathscr{0}$ & $\mathcal{U}$ & & J & & $\bar{J}$ & U & & ชै & J & $\mathscr{0}$ & 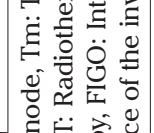 \\
\hline $\begin{array}{l}\text { क्ष } \\
\text { कँ }\end{array}$ & $\mathbb{s}$ & $\mathbb{s}$ & $\leq$ & $\underset{\Xi}{\Xi}$ & 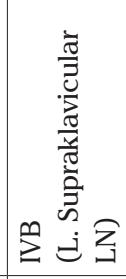 & 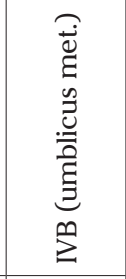 & $\unlhd$ & ঠ్ & 巳̂ & 巳一 & 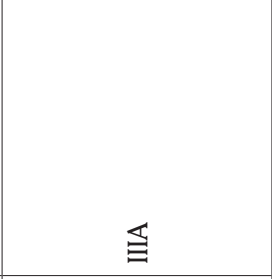 & 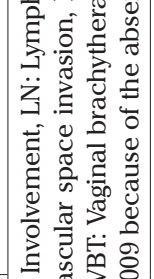 \\
\hline$\underset{\mathrm{g}}{\stackrel{0}{\mathrm{Z}}}$ & 涵 & 脐 & 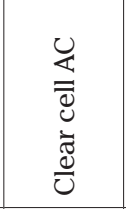 & 差 & 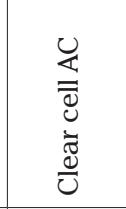 & 矛 & 矛 & 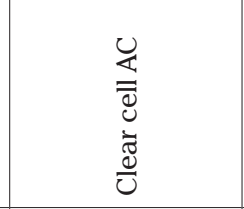 & 矛 & 预 & 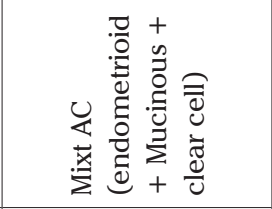 & 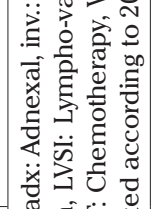 \\
\hline$\dot{4}$ & 18 & 1 & $\notin$ & 8 & 8 & $\stackrel{\infty}{\infty}$ & 오 & $\overline{0}$ & 8 & $\stackrel{1}{\circ}$ & 요 & \\
\hline 离 & - & - & - & $\sim$ & $\infty$ & $\sigma$ & L 18 & 0 & $\sim$ & $\infty$ & $\sigma$ & $\bar{\tau} \bar{c}$ \\
\hline & 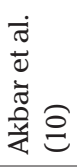 & 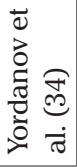 & & & & & & 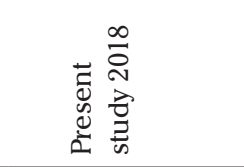 & & & & 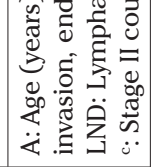 \\
\hline
\end{tabular}


Table 2. Features of the entire cohort

\begin{tabular}{|c|c|c|c|}
\hline \multicolumn{2}{|l|}{ Findings } & \multirow{2}{*}{$\begin{array}{l}\mathbf{n} \\
17\end{array}$} & \multirow{2}{*}{$\begin{array}{l}\% \\
40.5\end{array}$} \\
\hline \multirow{16}{*}{ Stage } & I & & \\
\hline & IA & 6 & 14.3 \\
\hline & IB & 1 & 2.4 \\
\hline & US stage I & 10 & 23.8 \\
\hline & $\mathrm{II}^{\mathrm{a}}$ & 2 & 4.8 \\
\hline & III & 15 & 35.8 \\
\hline & IIIA & 1 & 2.4 \\
\hline & IIIB & 1 & 2.4 \\
\hline & IIIC & 7 & 16.7 \\
\hline & IIIC2 & 5 & 11.9 \\
\hline & US & 2 & 4.8 \\
\hline & US stage III & 6 & 14.3 \\
\hline & IV & 5 & 11.9 \\
\hline & IVB & 3 & 7.1 \\
\hline & US stage IV & 2 & 4.8 \\
\hline & UR & 3 & 7.1 \\
\hline \multirow{8}{*}{$\begin{array}{l}\text { Histologic } \\
\text { type }\end{array}$} & Endometrioid & 13 & 31.0 \\
\hline & Grade 1 & 7 & 16.7 \\
\hline & Grade 2 & 3 & 7.1 \\
\hline & Grade 3 & 3 & 7.1 \\
\hline & Clear cell AC & 3 & 7.1 \\
\hline & AC (not specified) & 22 & 52.4 \\
\hline & $\begin{array}{l}\text { Mixed cell AC (grade } 3 \text { endometrioid + } \\
\text { mucinous }+ \text { clear cell) }\end{array}$ & 1 & 2.4 \\
\hline & UR & 3 & 7.1 \\
\hline \multirow{6}{*}{$\begin{array}{l}\text { Myometrial } \\
\text { invasion }^{\mathrm{b}}\end{array}$} & Confined to endometrium & 1 & 1.6 \\
\hline & Presence of myometrial invasion & 16 & 25.8 \\
\hline & Invasion $<1 / 2$ & 6 & 9.7 \\
\hline & Invasion $\geq 1 / 2$ & 9 & 14.5 \\
\hline & US & 1 & 1.6 \\
\hline & UR & 45 & 72.6 \\
\hline \multirow{17}{*}{$\begin{array}{l}\text { Site of } \\
\text { recurrent } \\
\text { peripheral } \\
\text { lymph node }^{\text {b }}\end{array}$} & Axillar & 4 & 6.4 \\
\hline & Right & 1 & 1.6 \\
\hline & Left & 1 & 1.6 \\
\hline & US & 2 & 3.2 \\
\hline & Inguinal & 26 & 41.9 \\
\hline & Right & 9 & 14.5 \\
\hline & Left & 10 & 16.1 \\
\hline & US & 7 & 11.3 \\
\hline & Supraclavicular & 16 & 25.9 \\
\hline & Right & 8 & 12.9 \\
\hline & Left & 4 & 6.5 \\
\hline & US & 4 & 6.5 \\
\hline & Cervical & 10 & 16.1 \\
\hline & Left & 3 & 4.8 \\
\hline & US & 7 & 11.3 \\
\hline & Cervical + supraclavicular & 5 & 8.1 \\
\hline & Axillar + supraclavicular & 1 & 1.6 \\
\hline
\end{tabular}

Table 2. Continued

\begin{tabular}{|c|c|c|c|}
\hline \multicolumn{2}{|l|}{ Findings } & \multirow{2}{*}{\begin{tabular}{|l|}
$\mathbf{n}$ \\
35 \\
\end{tabular}} & \multirow{2}{*}{$\begin{array}{ll}\% \\
83.3 \\
\end{array}$} \\
\hline \multirow{2}{*}{$\begin{array}{l}\text { Involvement } \\
\text { pattern }\end{array}$} & Isolated PNR & & \\
\hline & PNR with multiple involved sites & 7 & 16.7 \\
\hline \multirow{2}{*}{$\begin{array}{l}\text { Status of } \\
\text { the distant } \\
\text { recurrence } \\
\text { sites other } \\
\text { than PNR }\end{array}$} & Absent & 40 & 95.2 \\
\hline & Present & 2 & 4.8 \\
\hline \multirow{12}{*}{$\begin{array}{l}\text { Therapy } \\
\text { options at } \\
\text { recurrence }^{c}\end{array}$} & Radiotherapy + hormone therapy & 1 & 2.4 \\
\hline & Only chemotherapy & 5 & 11.9 \\
\hline & Chemotherapy + radiotherapy & 1 & 2.4 \\
\hline & Chemotherapy + hormone therapy & 1 & 2.4 \\
\hline & Only surgery & 2 & 4.8 \\
\hline & Surgery with adjuvant therapy & 13 & 31 \\
\hline & Surgery + radiotherapy & 6 & 14.3 \\
\hline & Surgery + chemotherapy & 5 & 11.9 \\
\hline & Surgery + chemo-radiotherapy & 1 & 2.4 \\
\hline & $\begin{array}{l}\text { Surgery + chemotherapy + } \\
\text { radiotherapy }\end{array}$ & 1 & 2.4 \\
\hline & Surgery + hormone therapy & 1 & 2.4 \\
\hline & UR & 2 & 4.7 \\
\hline \multirow{5}{*}{ End status } & AWOD & 16 & 38.1 \\
\hline & DOD & 18 & 42.9 \\
\hline & AWD & 2 & 4.8 \\
\hline & LFU & 3 & 7.1 \\
\hline & UR & 3 & 7.1 \\
\hline
\end{tabular}

PNR: Peripheral nodal recurrence; UR: Unreported; AWOD: Alive without disease; AWD: Alive with disease; LFU: Lost to follow-up; US: Unspecified, DOD: Dead of disease, a: Could not updated according to FIGO 2009 because of the absence of the involvement type of cervix, b: The distribution of the location analyzed among the 62 patients, c: 16 patients from report of the Foote et al. (19) were excluded because the therapy type was not given case by case

$(\mathrm{n}=1)$; retroperitoneal lymph nodes $(\mathrm{n}=2)$; and retroperitoneal lymph nodes together with involvement of the central pelvis $(n=1)$. In addition, two patients had distant organ metastasis (liver parenchyma with or without the tail of the pancreas) concurrent with PNR. Details of the features of recurrent disease are given in Table 2, 3 .

The rate of initial nodal involvement was higher in patients with inguinal PNR than patients with other sites of PNR [70\% $(7 / 10)$ vs $18.2 \%(2 / 11), p=0.03]$. The frequency of the presence of cervical invasion was higher in patients with PNR localized in the supraclavicular nodes than in patients with PNR sites besides the supraclavicular nodes $[100 \%(2 / 2)$ vs $12.5(2 / 16)$; $\mathrm{p}=0.039]$.

In $16(39.2 \%)$ patients, surgery was performed for the treatment of PNR. Seven (19.1\%) had non-surgical treatment, including chemotherapy $(\mathrm{n}=5)$, chemotherapy with radiotherapy $(\mathrm{n}=1)$, hormonal therapy with radiotherapy $(n=1)$ and hormonal 


\begin{tabular}{|c|c|c|c|c|c|c|c|c|c|c|}
\hline \multirow{2}{*}{ 兽 } & \multirow[b]{2}{*}{ 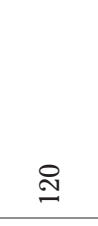 } & \multicolumn{7}{|c|}{ 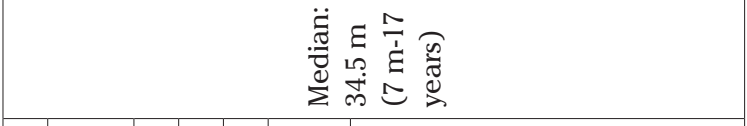 } & \multirow[b]{2}{*}{$\simeq$} & \multirow{2}{*}{ 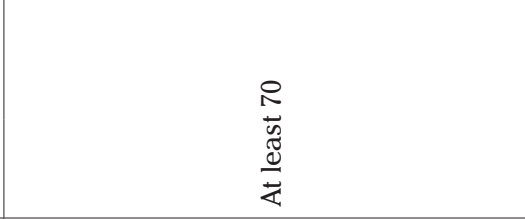 } \\
\hline & & 농 & 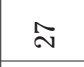 & 过 & $\bar{n}$ & i̊ & นึ & . & & \\
\hline 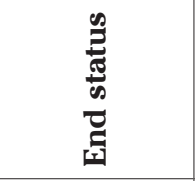 & 蛋 & 命 & 条 & 命 & 色 & $\begin{array}{l}0 \\
\vdots \\
\vdots\end{array}$ & ڤి & 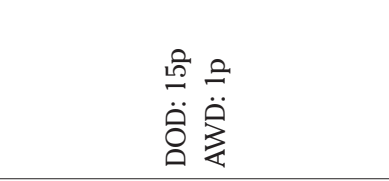 & ฏి & 蛋 \\
\hline 递泀 & & . & . & . & . & . & . & 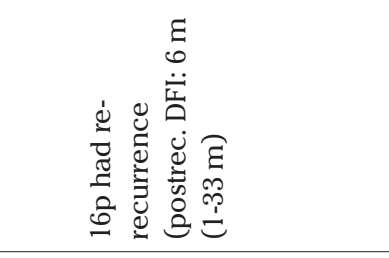 & & 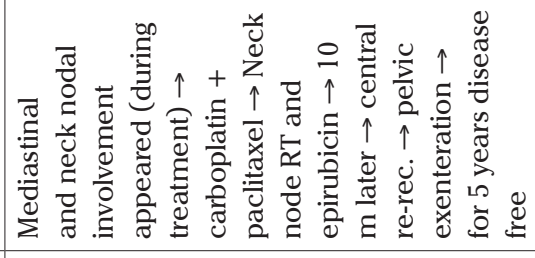 \\
\hline 危 & 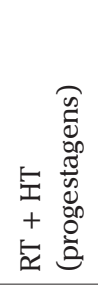 & 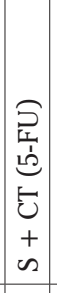 & 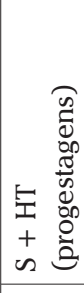 & 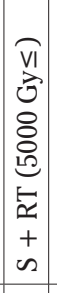 & 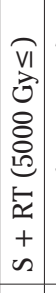 & 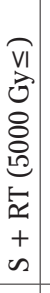 & 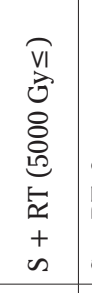 & 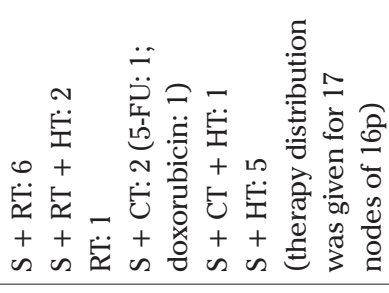 & 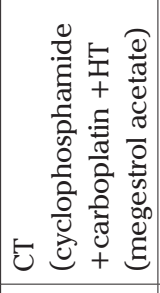 & 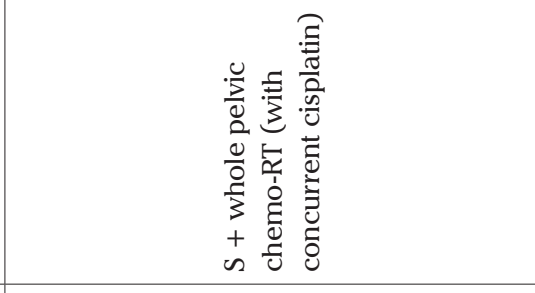 \\
\hline 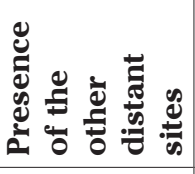 & $\stackrel{0}{z}$ & z & z & z & $z$ & $z$ & ż & z & z & $\dot{z}$ \\
\hline 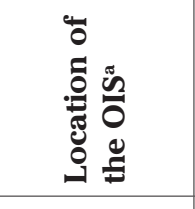 & ' & 1 & . & . & . & I & . & ' & 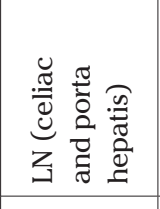 & 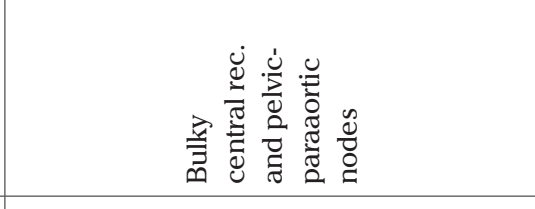 \\
\hline 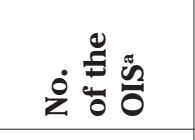 & 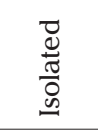 & 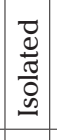 & 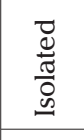 & 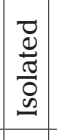 & 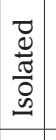 & 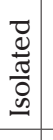 & 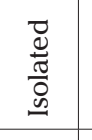 & 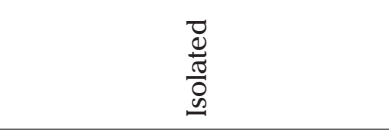 & 产 & $\frac{0}{2}$ \\
\hline 芦芯芯 & & $\sigma$ & $\sim$ & $\stackrel{\llcorner}{\rightarrow}$ & $\infty$ & $\nabla$ & $\infty$ & 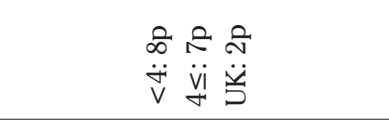 & 蓉 & $\stackrel{\Omega}{s}$ \\
\hline 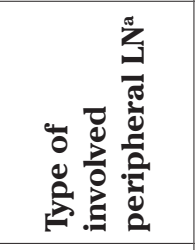 & $\frac{\overrightarrow{0}}{\bar{\pi}}$ & 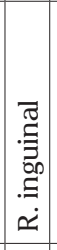 & 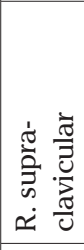 & 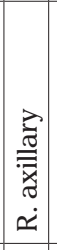 & 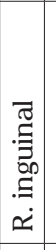 & 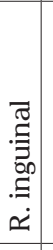 & 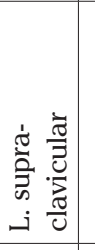 & 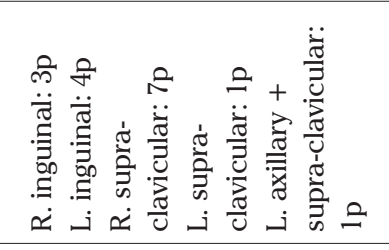 & 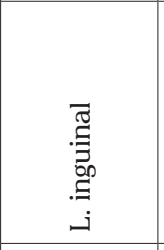 & 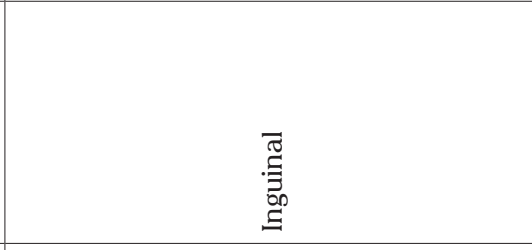 \\
\hline نُ & 密 & 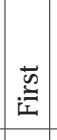 & 兽 & $\overrightarrow{0}$ & 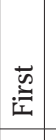 & 离 & 总 & 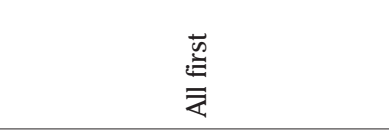 & 窟 & 总 \\
\hline 芯 & - & - & $\sim$ & $\infty$ & 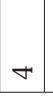 & L 18 & 0 & है & - & - \\
\hline & 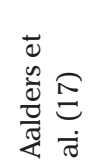 & & & & & & & 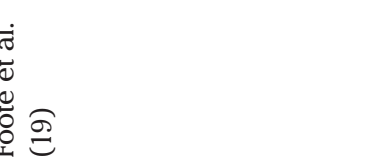 & 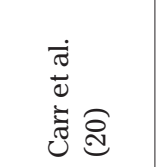 & 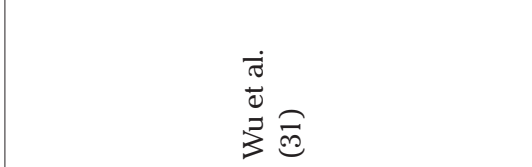 \\
\hline
\end{tabular}




\begin{tabular}{|c|c|c|c|c|c|c|c|c|c|c|}
\hline 导 & $\bar{\sim}$ & $\stackrel{\text { 号 }}{ }$ & $\mathscr{F}$ & $\stackrel{\infty}{\stackrel{+}{+}}$ & 占 & 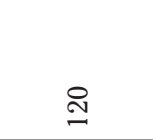 & ని & 芯 & ட̊ & $\stackrel{8}{q}$ \\
\hline 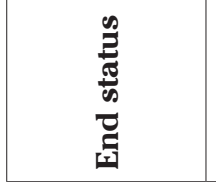 & 冬 & $\stackrel{\mathfrak{s}}{\mathfrak{s}}$ & ๐ิ & 命 & $\stackrel{\mathfrak{s}}{5}$ & ऐo & 命 & 嘀 & 岳 & ชิ \\
\hline 递 & . & . & 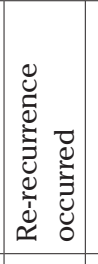 & . & . & . & . & . & & 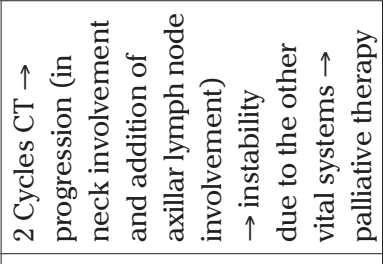 \\
\hline 苞 & 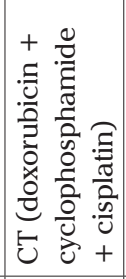 & $\stackrel{\mathfrak{s}}{\mathfrak{s}}$ & $\begin{array}{l}\text { tr } \\
+ \\
\text { is }\end{array}$ & $n$ & 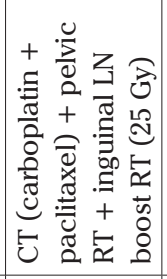 & 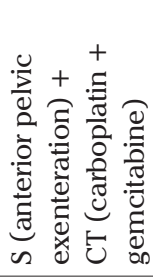 & 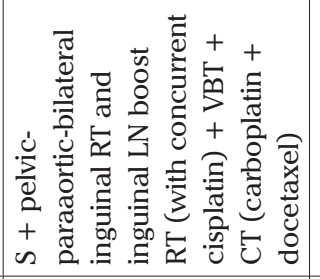 & 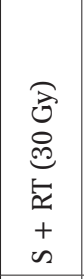 & 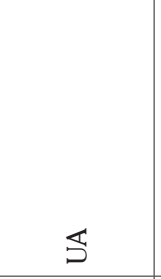 & 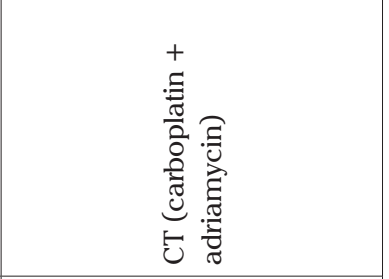 \\
\hline 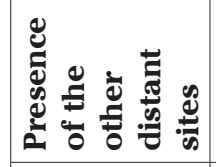 & z & z & $\stackrel{0}{z}$ & $\stackrel{0}{z}$ & $\stackrel{0}{z}$ & $\stackrel{0}{z}$ & $\stackrel{2}{z}$ & $\stackrel{0}{z}$ & 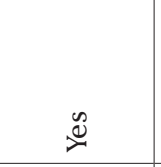 & z \\
\hline 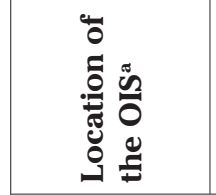 & . & . & , & . & , & 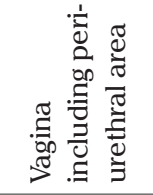 & 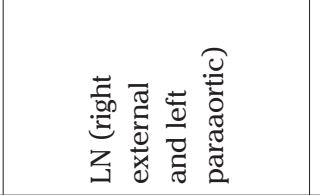 & & 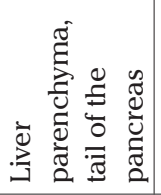 & . \\
\hline 之ं & 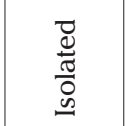 & 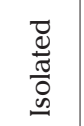 & 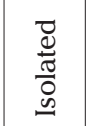 & 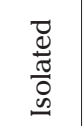 & 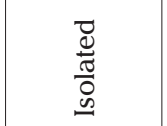 & 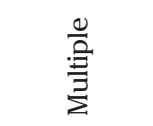 & $\frac{0}{\frac{0}{3}}$ & 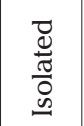 & $\frac{0}{\stackrel{0}{Z}}$ & 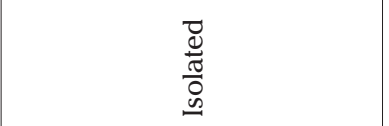 \\
\hline 芯 & $\stackrel{*}{*}$ & 号 & 箩 & $\stackrel{\text { g }}{\mathrm{s}}$ & 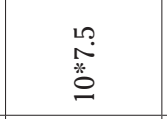 & \begin{tabular}{l}
$\stackrel{0}{\dot{*}}$ \\
\multirow{*}{*}{$\infty$} \\
$\stackrel{\infty}{+}$ \\
\end{tabular} & 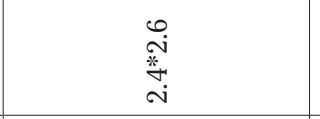 & $\stackrel{2}{*}$ & 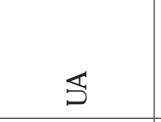 & 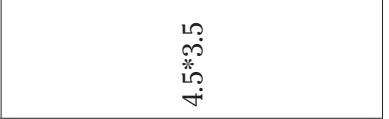 \\
\hline 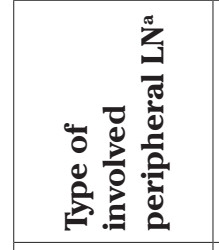 & 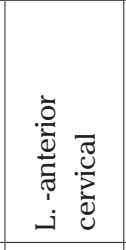 & 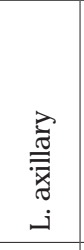 & 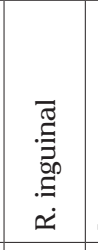 & 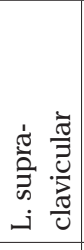 & 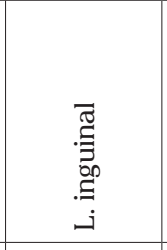 & 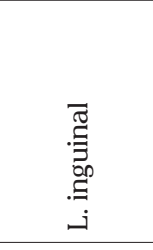 & 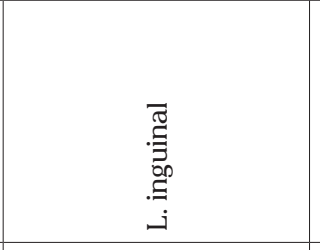 & 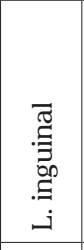 & 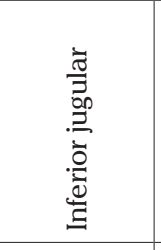 & 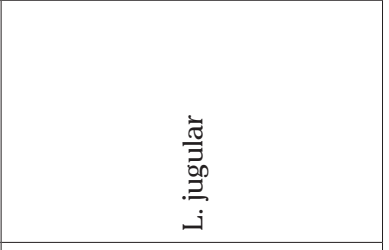 \\
\hline ن & 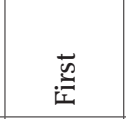 & 泀 & 蓓 & $\begin{array}{l}\bar{Z} \\
\tilde{0} \\
\dot{u} \\
n\end{array}$ & 哣 & 蒙 & 泀 & 蒂 & 范 & 蒙 \\
\hline \multirow[t]{2}{*}{ 岕 } & - & - & - & - & - & - & - & - & - & N \\
\hline & 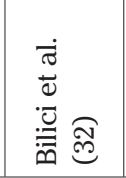 & 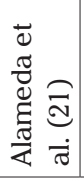 & 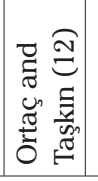 & 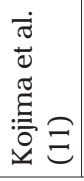 & 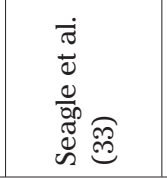 & 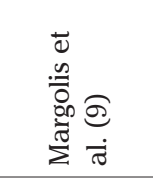 & 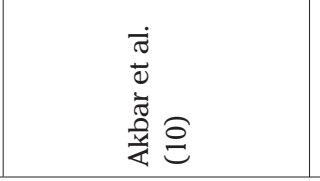 & 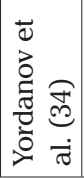 & \multicolumn{2}{|r|}{ 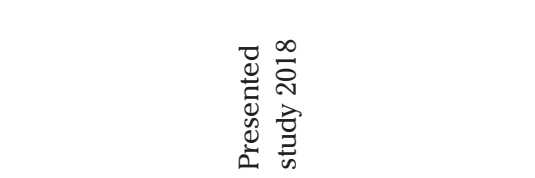 } \\
\hline
\end{tabular}




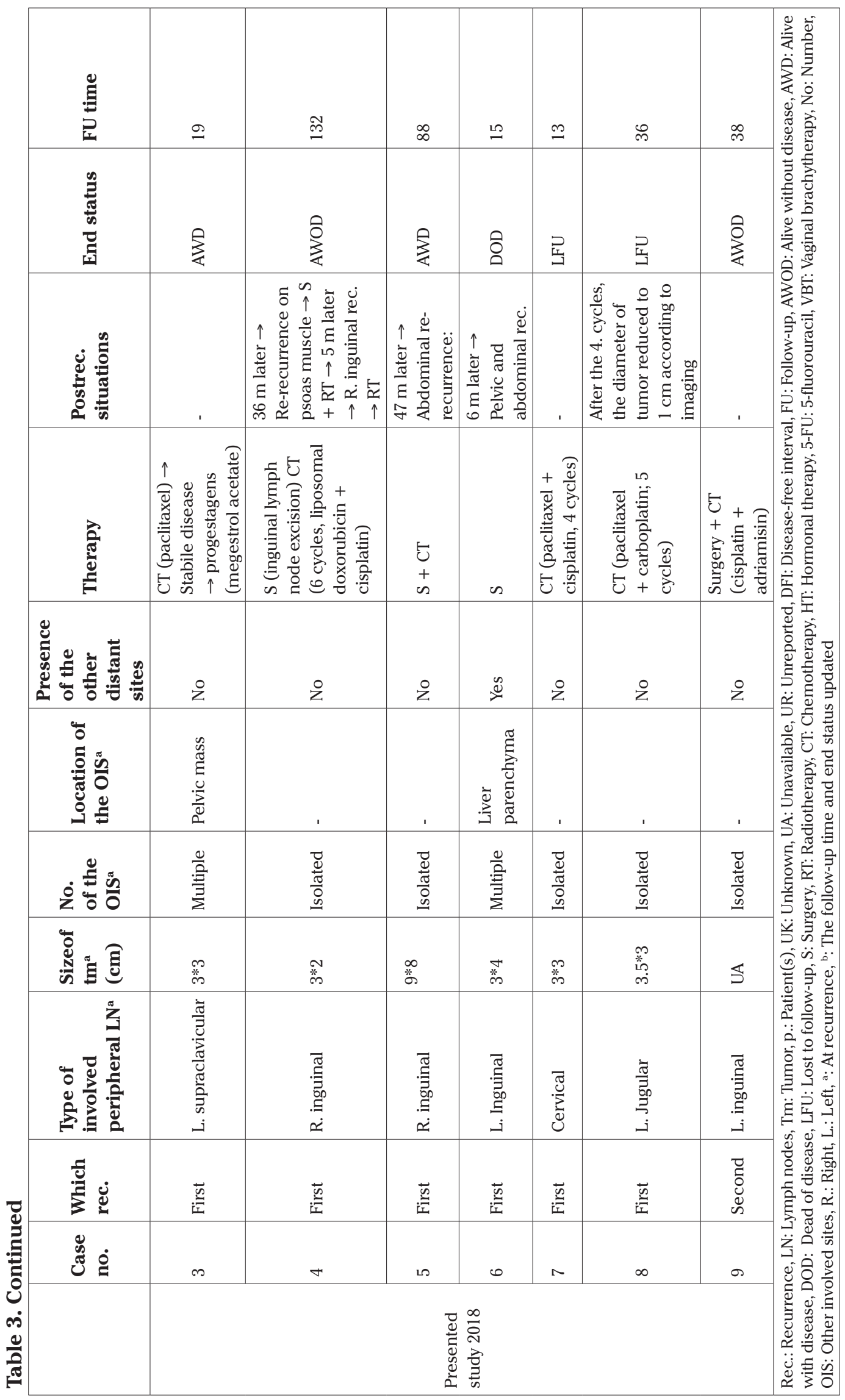


therapy with chemotherapy $(n=1)$. The treatment modality was unknown in two patients. The remaining 16 patients could not be grouped based on treatment modality because the type of therapy was not reported for each case so these patients were not included in the survival analysis (19).

The median (range) PRS was 22 (3-201) months. The 5-year and 10-year PRS were both 78\%. The median follow-up time was 45 (12-294) months. During follow-up, 18 patients dead of disease. In addition, two patients were alive with disease, 16 patients were alive without disease, three patients were lost to follow-up and the final status of three patients was not reported. In univariate analysis, the presence of distant hematogenous metastasis, as seen with PNR, was significantly associated with poor PRS $(p=0.005)$. The five-year PRS was $83 \%$ for patients who did not have distant hematogenous metastasis during PNR, whereas the patient who had distant hematogenous metastasis with PNR did not survive beyond 5 years (Figure 2). While the five-year PRS of the patients who had PNR with $>4 \mathrm{~cm}$ diameter was $50 \%$, all of those with $\leq 4 \mathrm{~cm}$ PNR survived passed 5 years $(p=0.09)$. Age, stage, histological type, DFI, the presence of recurrence before PNR, location or side of the recurrence, the diameter of the recurrent tumor, the presence of any other recurrences concurrent with PNR, and treatment types were not significantly associated with PRS. The relationship between clinico-pathological factors and PRS is shown in Table 4. Based on the analysis of the treatment options for isolated PNR ( $n=18)$, patients undergoing surgery had a 30\% higher 5-year PRS than those who did not undergo surgery. However, this difference was not significant (80\% vs $50 \% ; \mathrm{p}>0.05)$.

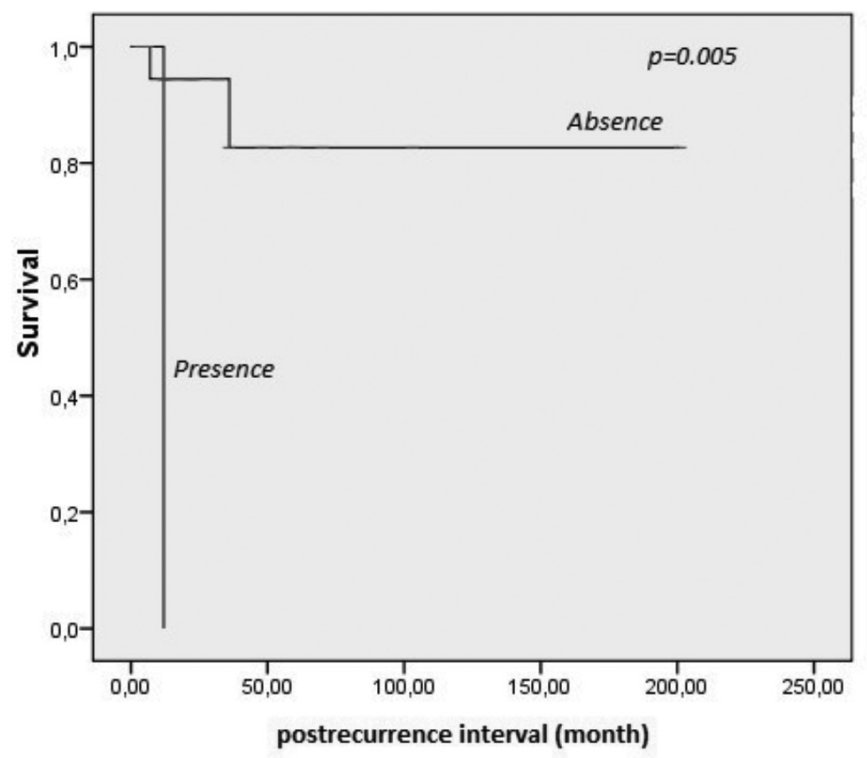

Figure 2. The presence of distant hematogenous metastasis, as seen with peripheral nodal recurrence, was significantly related to poor post-recurrence survival
Variables which were associated with a $\mathrm{p}<0.25$ in univariate analysis were tested in the multivariate analysis. The multivariate analysis model included tumor diameter $(>4 \mathrm{~cm}$ vs $\leq 4 \mathrm{~cm}$ ) and the presence of distant hematogenous metastasis coexisting with PNR (absent vs present). Multivariate analysis revealed that none of the variables was an independent prognostic factor for PRS (Table 5).

\section{Discussion}

The present study showed that the most common site of PNR were the inguinal lymph nodes. The major finding of our study was that concomitant hematogenous metastasis with PNR was related to poor PRS. Our study showed that no treatment options for PNR were superior to others.

Peripheral lymphatic failure is extremely rare in EC. The frequency of PNR was 1.92\% in all EC cases and 9.3\% among recurrent cases with EC (13). In our center, the frequency of PNR was $0.59 \%$ and $4.9 \%$ within the entire cohort and the group of patients with recurrent EC, respectively.

The most common lymphatic failure sites were the external iliac nodes (22). Kurra et al. (8) reported that the left supraclavicular lymph nodes are the most common distant lymphatic failure sites in EC. In our study, the most common site of PNR was the inguinal lymph nodes. The mechanisms underlying PNR remain unclear. One of the major mechanisms is thought to be the flow of tumoral cells via the thoracic duct (8). Although this explains tumor spread to the supraclavicular area, it cannot account for the inguinal nodal involvement in EC. Carr et al. (20) suggested that unopposed estrogen can cause proliferation of tumor cells in the lymphatic channels of the round ligament. However, only one of the cases with inguinal recurrence had a history of unopposed estrogen based on our literature review. The other hypothesis for isolated PNR is that there is a possibility of missing a metastasis due to the poor value of preoperative imaging in the detection of inguinal micrometastasis, especially for advanced disease (10). There is also a lower rate of detection of micrometastasis on initial evaluation of the retroperitoneal lymph nodes for early stages. Foote et al. (19) reported that the five-year PRS was 12\% for patients with isolated PNR. In our analysis, the five-year PRS was $78 \%$. One of the most likely reasons for the higher survival rate could be the advances in imaging that help in the early detection of recurrence and the high detection rate of metastases in other sites. The factors related to the prognoses of distant recurrences in EC vary (22-26). Only the presence of concomitant distant recurrence with PNR was associated with poor prognosis in PNR, although none of the factors affect the prognosis independently, according to our analysis.

A wide range of options exists for PNR treatment, including local excision, pelvic exenteration, chemotherapy, and 
Table 4. The relation between clinico-pathologic factors and post-recurrence survival

\begin{tabular}{|c|c|c|c|c|}
\hline & & $\mathbf{n}$ & 5-year PRS (\%) & $\mathbf{p}$ \\
\hline \multirow{2}{*}{ Age $^{\mathrm{a}, \mathrm{b}}$ (years) } & $<60$ & 10 & 89 & \multirow{2}{*}{0.186} \\
\hline & $\geq 60$ & 6 & 75 & \\
\hline \multirow{2}{*}{ Stage } & $1 \& 2$ & 6 & 67 & \multirow{2}{*}{0.890} \\
\hline & $3 \& 4$ & 15 & 83 & \\
\hline \multirow{2}{*}{ Histologic type ${ }^{a}$} & Endometrioid & 10 & 86 & \multirow{2}{*}{0.577} \\
\hline & Non-endometrioid & 3 & 67 & \\
\hline \multirow{2}{*}{ DFI (months) $)^{\mathrm{b}}$} & $<15$ & 9 & 44 & \multirow{2}{*}{0.339} \\
\hline & $\geq 15$ & 12 & 90 & \\
\hline \multirow{2}{*}{ Presence of the rec. before PNR } & Absent (first rec.) & 20 & 77 & \multirow{2}{*}{0.622} \\
\hline & Present (second rec.) & 2 & 100 & \\
\hline \multirow{2}{*}{ Site of recurrence } & Inguinal & 12 & 76 & \multirow{2}{*}{0.952} \\
\hline & Others & 10 & 86 & \\
\hline \multirow{2}{*}{ Recurrence site } & Right & 8 & 75 & \multirow{2}{*}{0.453} \\
\hline & Left & 11 & 78 & \\
\hline \multirow{2}{*}{ Diameter of the tumor at recurrence ${ }^{b}$} & $<4 \mathrm{~cm}$ & 10 & 100 & \multirow{2}{*}{0.090} \\
\hline & $\geq 4 \mathrm{~cm}$ & 8 & 50 & \\
\hline \multirow{2}{*}{ Presence of multiple involved sites during PNR } & Isolated PNR & 17 & 77 & \multirow{2}{*}{0.784} \\
\hline & PNR with multiple involved sites & 5 & 80 & \\
\hline \multirow{2}{*}{$\begin{array}{l}\text { Presence of the concomitant distant hematogenous } \\
\text { metastasis during PNR }\end{array}$} & Absent & 21 & 83 & \multirow{2}{*}{$0.005^{*}$} \\
\hline & Present & 1 & None & \\
\hline \multirow{9}{*}{ Therapy options at recurrence } & Surgery vs no surgery & & & \\
\hline & Surgery & 16 & 80 & \multirow{2}{*}{0.299} \\
\hline & No surgery & 6 & 67 & \\
\hline & \multicolumn{4}{|l|}{ CT absent vs CT present } \\
\hline & CT absent & 10 & 60 & \multirow{2}{*}{0.525} \\
\hline & CT present & 12 & 88 & \\
\hline & \multicolumn{4}{|l|}{ RT absent vs RT present } \\
\hline & RT absent & 13 & 80 & \multirow{2}{*}{0.584} \\
\hline & RT present & 9 & 75 & \\
\hline
\end{tabular}

Table 5. Multivariate analysis of factors predicting post-recurrence survival after peripheral nodal recurrence

\begin{tabular}{|c|c|c|}
\hline & Hazard ratio $(95 \% \mathrm{CI})$ & $\mathbf{p}$ \\
\hline \multicolumn{3}{|l|}{ Model } \\
\hline Diameter of the tumor at recurrence $(<4 \mathrm{~cm}$ vs $\geq 4 \mathrm{~cm})$ & $285164.3(0.001-\ldots)$ & 0.973 \\
\hline Presence of concomitant distant hematogenous metastasis during PNR (absent vs present) & $6.4(0.405-103.8)$ & 0.187 \\
\hline
\end{tabular}

radiotherapy. Treatment may also include a combination of these therapies and palliative therapy. Unfortunately, there are still no accepted criteria to aid in choosing the type of therapy for PNR. Surgical resection has an important value in isolated distant recurrence of $\mathrm{EC}$, and the probability of achieving complete resection is an important consideration in choosing surgery (24,26-28). However, based on recent knowledge, the necessity of multimodal therapies, especially systemic therapy, cannot be applicable, even for patients with negative margins following complete resection (29). In our study, no specific 
treatment had prognostic or survival superiority over any other. Therefore, the management approach in PNR is still at the discretion of the physician and also dependent upon patient preference. However, although not statistically significant, our results indicate that surgery could provide some survival advantage. Therefore, surgical treatment should be kept in the forefront as one component of treatment for isolated PNR. Similar to the interval of onset of other EC recurrences (29-33), $80 \%$ of PNR appeared in the first three years. However, PNR can develop as late as 23 years after initial diagnosis (34). Furthermore, a considerable number of patients had stage I disease (40.5\%) at initial diagnosis and developed PNR as their first recurrence. Therefore, long-term, close follow-up is critical for early diagnosis.

\section{Study limitation}

One of the limitations of the study is its retrospective design. Due to the differences in treatment approaches such as various doses of therapy, chemotherapeutic agents, radiotherapy equipment used, and surgical techniques, distinct conclusions cannot be drawn about outcomes of therapy. Although the other limitation appears to be a small sample size, our study included a relatively large sample of patients with PNR, which results from an extremely rare failure of EC. As far as we know, this is the first and largest study to evaluate factors associated with survival following peripheral nodal failures in EC patients.

\section{Conclusion}

Peripheral lymphatic failure was frequently localized in the inguinal lymph nodes. A concurrent distant hematogenous metastasis was the only factor related to poor survival. A wide range of therapies exists but none of the therapies appear more advantageous than any other. However, surgery can provide a survival benefit in patients who have isolated PNR. Further large-scale studies are needed to make definitive conclusions regarding treatment options.

Ethics Committee Approval: The study was approved by the Ethical Committee of the University of Health Sciences Turkey, Etlik Zübeyde Hanım Women's Health Training and Research Hospital (approval number: 47502, date: 25.06.2018).

Informed Consent: All patients signed an informed consent that allows the institution to use their clinical data.

\section{Peer-review: Externally peer-reviewed.}

Author Contributions: Surgical and Medical Practices: F.K., G.K.C., S.A., S.T., T.T., O.T., F.O., T.Tur.; Concept: F.K., G.K.C., C..K., N.T., T.T., O.T., T.Tur.; Design: F.K., G.K.C., S.T., O.T., F.O.,
T.Tur.; Data Collection or Processing: S.A., Ç.K., M.Ü., S.T., T.T., C.Ç., D.Y., N.T.; Analysis or Interpretation: F.K., G.K.C., M.Ü., T.T., O.T., F.O., T.Tur.; Literature Search: S.A., Ç.K., C.Ç., D.Y., M.Ü.; Writing: F.K., G.K.C., T.Tur.

Conflict of Interest: No conflict of interest is declared by the authors.

Financial Disclosure: The authors declared that this study received no financial support.

\section{References}

1. Jemal A, Bray F, Center MM, Ferlay J, Ward E, Forman D. Global cancer statistics. CA Cancer J Clin 2011; 61: 69-90.

2. Fung-Kee-Fung M, Dodge J, Elit L, Lukka H, Chambers A, Oliver T; Cancer Care Ontario Program in Evidence-based Care Gynecology Cancer Disease Site Group. Follow-up after primary therapy for endometrial cancer: a systematic review. Gynecol Oncol 2006; 101: 520-9.

3. Gadducci A, Cosio S, Fabrini MG, Fanucchi A, Barsotti C, Cristofani R, et al. Patterns of failures in endometrial cancer: clinicopathological variables predictive of the risk of local, distant and retroperitoneal failure. Anticancer Res 2011; 31: 3483-8.

4. Ben Arie A, Lavie O, Gdalevich M, Voldarsky M, Barak F, Schneider $\mathrm{D}$, et al. Temporal pattern of recurrence of stage I endometrial cancer in relation to histological risk factors. Eur J Surg Oncol 2012; 38: 166-9.

5. Mariani A, Webb MJ, Keeney GL, Aletti G, Podratz KC. Predictors of lymphatic failure in endometrial cancer. Gynecol Oncol 2002; 84: 437-42.

6. Vargo JA, Kim H, Houser CJ, Berhane H, Sukumvanich P, Olawaiye $\mathrm{AB}$, et al. Definitive salvage for vaginal recurrence of endometrial cancer: the impact of modern intensity-modulated-radiotherapy with image-based HDR brachytherapy and the interplay of the PORTEC 1 risk stratification. Radiother Oncol 2014; 113: 126-31.

7. Gadducci A, Guerrieri ME, Cosio S, Fabrini MG, Laliscia C, Attianese $\mathrm{D}$, et al. Rates, sites and times of recurrence and clinical outcome of endometrial cancer patients with histologically-positive nodes: an Italian two-center retrospective study. Anticancer Res 2018; 38: 1695-703.

8. Kurra V, Krajewski KM, Jagannathan J, Giardino A, Berlin S, Ramaiya $\mathrm{N}$. Typical and atypical metastatic sites of recurrent endometrial carcinoma. Cancer Imaging 2013; 13: 113-22.

9. Margolis B, Kim SW, Chi DS. Long-term survival after anterior pelvic exenteration and total vaginectomy for recurrent endometrial carcinoma with metastatic inguinal nodes at the time of surgery. Gynecol Oncol Rep 2017; 19: 39-41.

10. Akbar SA, Tunio MA, AlShakweer W, AlObaid A, AlAsiri M. Inguinal lymph node presenting as the delayed site of metastasis in early stage endometrial carcinoma: case report. Int J Surg Case Rep 2017; 32: 12-5.

11. Kojima M, Yokoyama J, Ito S, Ohba S, Fujimaki M, Ikeda K. Impact of middle and lower jugular neck dissection on supraclavicular lymph node metastasis from endometrial carcinoma. World J Surg Oncol 2012; 10: 143.

12. Ortaç F, Taşkın S. Inguinal recurrence of early stage endometrial cancer after 7 months of surgical staging: the role of PET-CT in diagnosis and management. Int J Clin Oncol 2012; 17: 283-5.

13. Salazar OM, Feldstein ML, DePapp EW, Bonfiglio TA, Keller BE, Rubin P, et al. Endometrial carcinoma: analysis of failures with 
special emphasis on the use of initial preoperative external pelvic radiation. Int J Radiat Oncol Biol Phys 1977; 2: 1101-7.

14. Shimamoto K, Saito T, Okadome M, Shimokawa M. Prognostic significance of the treatment-free interval in patients with recurrent endometrial cancer. Eur J Obstet Gynecol Reprod Biol 2014; 175: 92-6.

15. Long RT, Sala JM, Spratt JS. Endometrial carcinoma recurring after hysterectomy. A study of 64 cases, with observations on effective treatment modalities and implications for alteration of primary therapy. Cancer 1972; 29: 318-21.

16. Chung HH, Kang WJ, Kim JW, Park NH, Song YS, Chung JK, et al The clinical impact of [(18)F]FDG PET/CT for the management of recurrent endometrial cancer: correlation with clinical and histological findings. Eur J Nucl Med Mol Imaging 2008; 35: 1081-8.

17. Aalders JG, Abeler V, Kolstad P. Stage IV endometrial carcinoma: a clinical and histopathological study of 83 patients. Gynecol Oncol 1984; 17: 75-84.

18. Pecorelli S. Revised FIGO staging for carcinoma of the vulva, cervix, and endometrium. Int J Gynaecol Obstet 2009; 105: 103-4.

19. Foote RL, Schray MF, Wilson TO, Malkasian GD. Isolated peripheral lymph node recurrence of endometrial carcinoma. Cancer 1988 61: 2561-5.

20. Carr JA, Schoon PA, Look KY. An atypical recurrence of endometrial carcinoma following estrogen replacement therapy. Gynecol Oncol 1996; 60: 498-9.

21. Alameda F, Pijuan L, Lloveras B, Romero E, Carreras R, Serrano S. Axillary metastasis in a patient with double neoplasia: a case report. Acta Cytol 2010; 54: 1133-5.

22. Sohaib SA, Houghton SL, Meroni R, Rockall AG, Blake P, Reznek RH. Recurrent endometrial cancer: patterns of recurrent disease and assessment of prognosis. Clin Radiol 2007; 62: 28-34.

23. Toptas T, Karalok A, Ureyen I, Tasci T, Erol O, Bozkurt S, et al. Liver recurrence in endometrial cancer: a multi-institutional analysis of factors predictive of postrecurrence survival. Clin Exp Metastasis 2016; 33: 707-15.

24. Adachi M, Mizuno M, Mitsui H, Kajiyama H, Suzuki S, Sekiya R, et al. The prognostic impact of pulmonary metastasectomy in recurrent gynecologic cancers: a retrospective single-institution study. Nagoya J Med Sci 2015; 77: 363-72.

25. Turan T, Ureyen I, Karalok A, Tasci T, Turkmen O, Kocak O, et al. Pulmonary recurrence in patients with endometrial cancer. J Chin Med Assoc 2016; 79: 212-20.

26. Dowdy SC, Mariani A, Bakkum JN, Cliby WA, Keeney GL, Podratz KC. Treatment of pulmonary recurrences in patients with endometrial cancer. Gynecol Oncol 2007; 107: 242-7.

27. Kimyon G, Turan T, Basaran D, Turkmen O, Karalok A, Tasci T, et al. Is neurosurgery with adjuvant radiotherapy an effective treatment modality in isolated brain involvement from endometrial cancer? From case report to analysis. Int J Gynecol Cancer 2017; 27: 315-25.

28. Dresler CM, Goldberg M. Surgical management of lung metastases: selection factors and results. Oncology (Williston Park) 1996; 10: 649-55.

29. Zanfagnin V, Ferrero A, Biglia N, Aletti G, Gill SE, Makdisi PB, et al. The role of surgery in recurrent endometrial cancer. Expert Rev Anticancer Ther 2016; 16: 741-50.

30. Creutzberg CL, Nout RA, Lybeert ML, Warlam-Rodenhuis CC, Jobsen JJ, Mens JW, et al. Fifteen-year radiotherapy outcomes of the randomized PORTEC-1 trial for endometrial carcinoma. Int J Radiat Oncol Biol Phys 2011; 81: e631-8.

31. Wu YC, Huang SL, Chuang CK, Jung SM, Lai CH. Successful salvage treatment of recurrent endometrial cancer with bulky central tumor and extensive lymph node metastasis. A case report. Eur J Gynaecol Oncol 2004; 25: 739-41.

32. Bilici A, Karci E, Altun E, Ozkara SK, Uygun K, Aksu G, et al. An unusual case of recurrent endometrial cancer presented with isolated cervical lymph node metastasis. Arch Gynecol Obstet 2009; 280: 153-6.

33. Seagle BL, Cleason DM, Samuelson R, Shahabi S. Inguinal node metastasis of low-grade endometrial endometrioid adenocarcinoma in a morbidly obese patient. Conn Med 2015; 79: 415-7.

34. Yordanov A, Karamanliev M, Strashilov S. Delayed inguinal site metastasis in early-stage endometrial cancer: a case report. Indian J Gynecol Oncol 2018; 16: 14. 